\title{
Wick products of complex valued random variables
}

\author{
Fred Espen Benth ${ }^{1}$, Bernt Øksendal ${ }^{2}$, Jan Ubøe ${ }^{3}$ and Tusheng Zhang ${ }^{3}$ \\ 1. Department of Mathematics V, University of Mannheim A5, D-68131 Mannheim, Ger- \\ many
}

2. Department of Mathematics, University of Oslo, Box 1053, Blindern

N-0316 Oslo 3, Norway

3. Department of Engineering, Stord/Haugesund College, Skåregaten 103

N-5 500 Haugesund, Norway

\begin{abstract}
In this paper we consider Wick products of complex valued random variables. We prove that Wick products of such variables coincide with the ordinary product in a variety of cases. Ordinary SDEs are considered in relation to their Wick versions. We present examples where these notions are equivalent in the complex case.
\end{abstract}

\section{Introduction}

The relationship between stochastic integration and complex analysis has been a topic of several authors. Analytic functions are conformal mappings and they will always map Brownian paths into new Brownian paths. The area is thus characterized by a number of phenomena which do not appear in the real case. The basis for many of these issues can be found from the complex version of the Ito formula, see e.g. [14]:

$$
d f\left(Z_{t}\right)=\frac{\partial f}{\partial z} d Z_{t}+\frac{\partial f}{\partial \bar{z}} d \bar{Z}_{t}+\frac{1}{2} \frac{\partial^{2} f}{\partial z^{2}} d Z_{t} d Z_{t}+\frac{\partial^{2} f}{\partial z \partial \bar{z}} d Z_{t} d \bar{Z}_{t}+\frac{1}{2} \frac{\partial^{2} f}{\partial \bar{z}^{2}} d \bar{Z}_{t} d \bar{Z}_{t}
$$

If $f$ happens to be an analytic function, this simplifies to:

$$
d f\left(Z_{t}\right)=\frac{\partial f}{\partial z} d Z_{t}+\frac{1}{2} \frac{\partial^{2} f}{\partial z^{2}} d Z_{t} d Z_{t}
$$

Usually one only wants to consider processes $Z_{t}$ with some kind of holomorphic structure, e.g. conformal martingales see [5] or [18]. In these cases the quadratic variation term $d Z_{t} d Z_{t}$ vanish, and we end up with the ordinary chain rule: 
F.E.Benth et al

$$
d f\left(Z_{t}\right)=\frac{\partial f}{\partial z} d Z_{t}
$$

Once we have a chain rule of this form, we are able to solve various problems in stochastic calculus using simple techniques from classical calculus. The awkward correction terms from the usual Ito calculus are no longer present, and the basic intuition from ordinary differential equation applies without change. By contrast, one can achieve more or less the same effect using Wick products and Wick calculus. For references to the theory of Wick calculus see [6], [8], [10] or [12]. This way of approach applies already in the real variable case.

\section{Some notation}

Let $S(\mathbb{R})$ be denote the usual Schwartz space of rapidly decreasing smooth $\left(C^{\infty}\right)$ functions on $\mathbb{R}$ with its dual space $S^{\prime}(\mathbb{R})$ equipped with the weak star topology, and let $S_{\mathbb{C}}^{\prime}(\mathbb{R})$ denote the complexification of $S^{\prime}(\mathbb{R})$. On $S_{\mathbb{C}}^{\prime}(\mathbb{R})$ we define a probability measure $\mu$ as the product of two white noise measures, see [7]. To be more precise, the complexification of the real white noise probability space is carried out as follows. Put:

$$
S_{\mathbb{C}}(\mathbb{R})=S(\mathbb{R})+i S(\mathbb{R}) \quad \text { and: } \quad s_{\mathbb{C}}^{\prime}(\mathbb{R})=S^{\prime}(\mathbb{R})+i S^{\prime}(\mathbb{R})
$$

By the Bochner-Minlos theorem, define two measures $\mu_{1}$ and $\mu_{2}$ on $S^{\prime}(\mathbb{R})$ with:

$$
\int_{S^{\prime}(\mathbb{R})} \exp (i\langle\omega, \phi\rangle) d \mu_{j}(\omega)=\exp \left(-\frac{1}{4}\|\phi\|_{L^{2}(\mathbb{R})}^{2}\right), \quad j=1,2 .
$$

With $\mathcal{B}$ the Borel $\sigma$-algebra on $S_{\mathbb{C}}^{\prime}(\mathbb{R})$, introduce the product measure $v=\mu_{1} \times \mu_{2}$. Then the triplet:

$$
\left(s_{\mathbb{C}}^{\prime}(\mathbb{R}), \mathcal{B}, v\right)
$$

is called the complex white noise probability space. From the expression (1.5) we get the familiar isometry $E\left[|<\cdot, \phi>|^{2}\right]=\|\phi\|_{L^{2}(\mathbb{R})}^{2}$ for all $\phi \in S(\mathbb{R})$ where $\langle\omega, \phi>=\omega(\phi)$ is the dual action. Using this isometry, we can define $\langle\omega, \phi\rangle:=\lim _{k \rightarrow \infty}\left\langle\omega, \phi_{k}\right\rangle$ for all $\phi \in L^{2}(\mathbb{R})\left(\phi_{k}\right.$ is any sequence in $S(\mathbb{R})$ s.t. $\phi_{k} \rightarrow \phi$ in $\left.L^{2}(\mathbb{R})\right)$. This allows us to define:

$$
\tilde{\mathbb{B}}_{t}(\omega):=\left\langle\omega, 1_{[0, t)}\right\rangle=\left\langle\omega_{\text {real }}, 1_{[0, t)}\right\rangle+i\left\langle\omega_{\text {imaginary }}, 1_{[0, t)}\right\rangle
$$

$\tilde{\mathbb{B}}_{t}$ is then essentially a Brownian motion in the complex plane in the sense that there exist a $t$-continuous version $\mathbb{B}_{t}$ of $\tilde{\mathbb{B}}_{t}$ such that $\mathbb{B}_{t}$ is a Brownian motion in the complex plane. We let $B_{1 t}$ and $B_{2 t}$ denote the real and the imaginary components of $\mathbb{B}_{t}$. We also 
need the corresponding white noise processes and indicate these as $\mathbb{W}_{t}, W_{1 t}$ etc. The familiar constructions of white noise analysis now carry over to the complex case with some minor modifications.

Following Hida [7], we introduce the complex Hermite polynomials $H_{n, m}(z, \bar{z})$ as:

$$
H_{n, m}(z, \bar{z})=(-1)^{n+m} \exp (z \bar{z}) \frac{\partial^{n+m}}{\partial \bar{z}^{n} \partial z^{m}} \exp (-z \bar{z})
$$

where $n, m$ are non-negative integers. With this definition, we see that our Brownian motion can be written:

$$
\mathbb{B}_{t}(\omega)=H_{1,0}\left(<\omega, 1_{[0, t)}>, \overline{<\omega, 1_{[0, t)}>}\right)
$$

Denote by $\left(L_{\mathbb{C}}^{2}\right):=L^{2}(v)$, and let $\mathcal{H}_{(n, m)}$ be the subspace spanned by the functions $\left\{H_{n, m}\left(<\omega, e_{i}>, \overline{\left.<\omega, e_{i}>\right)}\right\}_{i}\right.$, where $\left\{e_{i}\right\}_{i}$ is a CONS in $L_{\mathbb{C}}^{2}(\mathbb{R})$. We will make the assumption that $e_{i} \in S_{\mathbb{C}}(\mathbb{R})$ for all $i=1,2, \ldots$. From [7], proof of prop. 6.11, we have the orthogonality relation:

$$
\begin{aligned}
& \int_{S_{\mathbb{C}}^{\prime}(\mathbb{R})} H_{p, k}(<\omega, \psi>, \overline{\langle\omega, \psi>}) \overline{H_{n, m}(<\omega, \gamma>, \overline{<\omega, \gamma>})} d v(\omega) \\
= & \delta_{p, n} \delta_{q, m} p ! q !(\gamma, \psi)^{p} \overline{(\gamma, \psi)^{q}}
\end{aligned}
$$

In [7] it is shown that we have a Wiener-Ito-Segal decomposition for every $\phi \in\left(L_{\mathbb{C}}^{2}\right)$ :

$$
\phi(\omega)=\sum_{n, m} \phi_{n, m}(\omega)
$$

where $\phi_{n, m} \in \mathcal{H}_{(n, m)}$. We obtain a Fock space structure, i.e. $\phi$ is in a one-to-one correspondence with a sequence of functions $\left\{f^{(n, m)}\right\}_{n, m}$, with $f^{(n, m)} \in L_{\mathbb{C}}^{2}\left(\mathbb{R}^{n+m}\right)$. Moreover:

$$
\|\phi\|_{\left(L_{\mathbb{C}}^{2}\right)}^{2}=\sum_{n=1}^{\infty} \sum_{m=1}^{n} n ! m !\left|f^{(n, m)}\right|_{L_{\mathbb{C}}^{2}\left(\mathbb{R}^{n+m}\right)}^{2}
$$

We introduce the complex Kondratiev spaces of random test functions and distributions. Our construction follows closely the one found in [1]. Let $\mathbf{P}:=\mathbf{P}\left(\left\{e_{i}\right\}\right)$ be the space of polynomials as defined in [7], Ch. 6.3. Every element $\phi \in \mathbf{P}$ is expressible in the form:

$$
\phi(\omega)=\sum_{n=0}^{N} \sum_{m=0}^{n} \phi_{n, m}(\omega)
$$


where $\phi_{n, m} \in \mathcal{H}_{(n, m)}$. Let the space $\left(S_{\mathbb{C}}\right)_{p}^{1}$ be the completion of $\mathbf{P}$ in the norm:

$$
\|\phi\|_{2, p, \mathbb{C}}^{2}=\sum_{n=0}^{\infty} \sum_{m=0}^{n}(n ! m !)^{2}\left|f^{(n, m)}\right|_{2, p, \mathbb{C}}^{2}
$$

where $|\cdot|_{2, p, \mathbb{C}}$ is the complexification of the norm $|\cdot|_{2, p}:=\left|A^{p} \cdot\right|_{2} . A$ is the harmonic oscillator. The complex Kondratiev space of random test functions is the projective limit of the spaces $\left(S_{\mathbb{C}}\right)_{p}^{1}$, and is denoted $\left(S_{\mathbb{C}}\right)^{1}$. Its dual, the space of complex Kondratiev distributions, is denoted $\left(S_{\mathbb{C}}\right)^{-1}$. All elements $\Phi \in\left(S_{\mathbb{C}}\right)^{-1}$ is in a one-to-one correspondence with a sequence of functions $\left\{F^{(n, m)}\right\}_{n, m}$, with $F^{(n, m)} \in S_{\mathbb{C}}^{\prime}\left(\mathbb{R}^{n+m}\right)$, such that for a $p>0$ :

$$
\|\Phi\|_{-2,-p, \mathbb{C}}^{2}:=\sum_{n=0}^{\infty} \sum_{m=0}^{n}\left|F^{(n, m)}\right|_{2,-p, \mathbb{C}}^{2}<\infty
$$

On the space of complex Kondratiev distributions, we introduce the $S$-transform: For $\Phi \in\left(S_{\mathbb{C}}\right)^{-1}$ and $\xi \in S_{\mathbb{C}}(\mathbb{R})$, let:

$$
S \Phi(\xi):=\left\langle\bar{\Phi}, \exp \left(<\cdot, \xi>+\overline{<\cdot, \xi>}-|\xi|_{2}^{2}\right)\right\rangle
$$

where $\langle\cdot, \cdot\rangle$ is the dual pairing between $\left(S_{\mathbb{C}}\right)^{-1}$ and $\left(S_{\mathbb{C}}\right)^{1}$. It is easy to see that for $\phi \in\left(L_{\mathbb{C}}^{2}\right)$ :

$$
S \phi(\xi)=\int_{S^{\prime}(\mathbb{R})} \overline{\phi(\omega)} \exp \left(<\omega, \xi>+\overline{<\omega, \xi>}-|\xi|_{2}^{2}\right) d v(\omega)
$$

From formula A.40 in [7], we have:

$$
\exp \left(<\omega, \xi>+\overline{<\omega, \xi>}-|\xi|_{2}^{2}\right)=\sum_{n, m=0}^{\infty} \frac{|\xi|_{2}^{n+m}}{n ! m !} H_{n, m}\left(<\omega, \xi \cdot|\xi|_{2}^{-1}>\overline{<\omega, \xi \cdot|\xi|_{2}^{-1}>}\right)
$$

Using the orthogonality relation for the complex Hermite polynomials, we obtain:

$$
S B_{t}(\xi)=|\xi|_{2}\left(1_{[0, t)}, \xi \cdot|\xi|_{2}^{-1}\right)=\int_{0}^{t} \overline{\xi(s)} d s
$$

From [1] it is known that the $S$-transform characterizes the Kondratiev distributions. Consider a function $G: \mathcal{U} \rightarrow \mathbb{C}$, where $\mathcal{U}$ is a neighborhood around zero in $S_{\mathbb{C}}(\mathbb{R})$. If $G$ is locally bounded on $\mathcal{U}$, and the mapping $z \rightarrow G(\xi+z \eta)$ is analytic in a neighborhood around zero in $\mathbb{C}$ for each pair $\xi, \eta \in \mathcal{U}$, then there exists a $\Phi \in\left(S_{\mathbb{C}}\right)^{-1}$ such that $S \Phi=G$. 
Opposite, every element in $\left(S_{\mathbb{C}}\right)^{-1}$ has a $S$-transform which is of this type. We refer the reader to the papers [12] and [1], and the contribution of F. E. Benth in this volume, for more about the Kondratiev distribution space. The Wick product of two complex Kondratiev distributions is defined as follows: Let $\Phi, \Psi \in\left(S_{\mathbb{C}}\right)^{-1}$, then:

$$
\Phi \diamond \Psi=S^{-1}(S \Phi \cdot S \Psi)
$$

With this definition, we can easily calculate the $S$-transform of $\mathbb{B}_{t}^{\diamond k}$, for a integer $k$ :

$$
S \mathbb{B}_{t}^{\diamond k}(\xi)=\left(\int_{0}^{t} \overline{\xi(s)} d s\right)^{k}
$$

A straightforward calculation shows that:

$$
s\left(H_{k, 0}(<\omega, \xi>, \overline{<\omega, \xi>)})(\xi)=\left(\int_{0}^{t} \overline{\xi(s)} d s\right)^{k}\right.
$$

Hence, we find:

$$
\mathbb{B}_{t}^{\diamond k}(\omega)=H_{k, 0}\left(<\omega, 1_{[0, t)}>, \overline{\left\langle\omega, 1_{[0, t)}>\right.}\right)=<\omega, 1_{[0, t)}>^{k}=\mathbb{B}_{t}(\omega)^{k}
$$

\section{Complex Wick multiplication}

We now let $f(z)=\sum_{n=0}^{\infty} a_{n} z^{n}$ be an entire function. If $X$ is a random variable, we define the Wick version of $f(X)$ by the expression:

$$
f^{\diamond}(X)=\sum_{n=0}^{\infty} a_{n} X^{\diamond n}=\lim _{N \rightarrow \infty} \sum_{n=0}^{N} a_{n} X^{\diamond n}
$$

the limit being taken in $\left(S_{\mathbb{C}}\right)^{-1}$, see [10] or [12]. If $X \in\left(S_{\mathbb{C}}\right)^{-1}$, this limit always exists. With these conventions the following theorem follows trivially from (1.23).

\section{THEOREM 2.1}

Let $f: \mathbb{C} \rightarrow \mathbb{C}$ be an entire function, and let $f^{\diamond}$ denote the Wick version. Then:

$$
f\left(\mathbb{B}_{t}\right)=f^{\diamond}\left(\mathbb{B}_{t}\right)
$$


We have proved that Wick-powers of complex Brownian motion coincide with usual powers. In the following we want to extend this property to other random variables as well. We first observe the following lemma:

LEMMA 2.2

$$
\mathbb{B}_{s}^{\diamond m} \diamond \mathbb{B}_{t}^{\diamond n}=\mathbb{B}_{s}^{m} \cdot \mathbb{B}_{t}^{n}
$$

\section{PROOF}

Assume that $t \geq s$, then we have:

$$
\begin{aligned}
\mathbb{B}_{s}^{\diamond m} \diamond \mathbb{B}_{t}^{\diamond n} & =\mathbb{B}_{s}^{\diamond m} \diamond\left(\mathbb{B}_{t}-\mathbb{B}_{s}+\mathbb{B}_{s}\right)^{\diamond n} \\
& =\mathbb{B}_{s}^{\diamond m} \diamond \sum_{k=0}^{n}\left(\begin{array}{l}
n \\
k
\end{array}\right)\left(\mathbb{B}_{t}-\mathbb{B}_{s}\right)^{\diamond k} \diamond \mathbb{B}_{s}^{\diamond(n-k)} \\
& =\sum_{k=0}^{n}\left(\begin{array}{l}
n \\
k
\end{array}\right)\left(\mathbb{B}_{t}-\mathbb{B}_{s}\right)^{\diamond k} \diamond \mathbb{B}_{s}^{\diamond(n-k+m)} \\
& =\sum_{k=0}^{n}\left(\begin{array}{l}
n \\
k
\end{array}\right)\left(\mathbb{B}_{t}-\mathbb{B}_{s}\right)^{k} \diamond \mathbb{B}_{s}^{n-k+m} \\
& =\sum_{k=0}^{n}\left(\begin{array}{l}
n \\
k
\end{array}\right)\left(\mathbb{B}_{t}-\mathbb{B}_{s}\right)^{k} \cdot \mathbb{B}_{s}^{n-k+m} \\
& =\mathbb{B}_{s}^{m} \cdot \mathbb{B}_{t}^{n}
\end{aligned}
$$

In the fifth equality we have used that $\mathbb{B}_{t}-\mathbb{B}_{s}$ and $\mathbb{B}_{s}$ are strongly independent. In this case the Wick product always coincide with the ordinary product, see [6].

\section{PROPOSITION 2.3}

Let $p$ be a polynomial in $k$ complex variables, then:

$$
p^{\diamond}\left(\mathbb{B}_{t_{1}}, \mathbb{B}_{t_{2}}, \ldots, \mathbb{B}_{t_{k}}\right)=p\left(\mathbb{B}_{t_{1}}, \mathbb{B}_{t_{2}}, \ldots, \mathbb{B}_{t_{k}}\right)
$$

where $p^{\diamond}$ is interpreted in the sense that all powers are Wick powers.

\section{PROOF}

Use lemma 1.4 repeatedly to see that:

$$
\mathbb{B}_{t_{1}}^{\diamond n_{1}} \diamond \mathbb{B}_{t_{2}}^{\diamond n_{2}} \diamond \cdots \diamond \mathbb{B}_{t_{k}}^{\diamond n_{k}}=\mathbb{B}_{t_{1}}^{n_{1}} \cdot \mathbb{B}_{t_{2}}^{n_{2}} \cdots \mathbb{B}_{t_{k}}^{n_{k}}
$$

The general result then follows by linearity. 
For easy reference we will call any expression on the form $\Pi_{k}=p\left(\mathbb{B}_{t_{1}}, \mathbb{B}_{t_{2}}, \ldots, \mathbb{B}_{t_{k}}\right)$ a $\mathbb{B}$-analytic polynomial. The multiplicative property (2.4) can now be extended to limits of $\mathbb{B}$-analytic polynomials. A convenient space to work in is then the space $\left(S_{\mathbb{C}}\right)^{-1}$ of Kondratiev distributions, see [10] or [12]. We start out with some definitions:

\section{DEFINITION 2.4}

$X \in\left(S_{\mathbb{C}}\right)^{-1}$ is called $\mathbb{B}$-analytic if there exists a sequence $X_{n}$ of $\mathbb{B}$-analytic polynomials such that $X_{n} \rightarrow X$ strongly in $\left(S_{\mathbb{C}}\right)^{-1}$.

\section{DEFINITION 2.5}

$X \in\left(L^{p}\right), p>1$, is $\mathbb{B}_{p}$-analytic if there exists a sequence $X_{n}$ of $\mathbb{B}$-analytic polynomials such that $X_{n} \rightarrow X$ in $\left(L^{p}\right)$.

From these definitions, we have:

COROLLARY 2.6

Let $X \in\left(L^{p}\right), p>1$, be $\mathbb{B}_{p}$-analytic. Then $X$ is $\mathbb{B}$-analytic.

PROOF

By assumption we have a sequence $X_{n}$ of $\mathbb{B}$-analytic polynomials converging to $X$ in $\left(L^{p}\right)$. But convergence in $\left(L^{p}\right)$ implies strong convergence in $\left(S_{\mathbb{C}}\right)^{-1}$, see [12]. Hence, $X$ is $\mathbb{B}$ analytic.

\section{COROLLARY 2.7}

If $\left\{X_{n}\right\}$ is a sequence of $\mathbb{B}$-analytic elements which converges strongly to $X$ in $\left(S_{\mathbb{C}}\right)^{-1}$, then $X$ is $\mathbb{B}$-analytic.

\section{PROOF}

The proof is straightforward: Since $X_{n}$ converges strongly to $X$ in $\left(S_{\mathbb{C}}\right)^{-1}$, there exists a $p>0$ such that:

$$
\left\|X_{n}-X\right\|_{2,-p, \mathbb{C}} \rightarrow 0
$$

For each $n$, let $\left\{Y_{n}^{m}\right\}$ be a sequence of $\mathbb{B}$-analytic polynomials converging strongly to $X_{n}$ in $\left(S_{\mathbb{C}}\right)^{-1}$. (Such a sequence exists by definition of $\mathbb{B}$-analyticity). Since $X_{n}$ is an element in $\left(S_{\mathbb{C}}\right)_{-p}^{-1}$, we have:

$$
\left\|Y_{n}^{m}-X_{n}\right\|_{2,-p, \mathbb{C}} \rightarrow 0, \quad m \rightarrow \infty
$$


F.E.Benth et al

This yields that for each $n$, there exists a natural number $N_{n}$ such that:

$$
\left\|Y_{n}^{m}-X_{n}\right\|_{2,-p, \mathbb{C}}<\frac{1}{n}, \quad \text { for } m \geq N_{n}
$$

It is then easy to see that the sequence $\left\{Y_{n}^{N_{n}}\right\}$ of $\mathbb{B}$-analytic polynomials converges strongly in $\left(S_{\mathbb{C}}\right)^{-1}$ to $X$ : For a given $\epsilon>0$, we find a $M_{\epsilon}$ such that $1 / n<\epsilon / 2$ and:

$$
\left\|X-X_{n}\right\|_{2,-p, \mathbb{C}}<\epsilon / 2
$$

for $n \geq M_{\epsilon}$. Hence, by the triangle inequality:

$$
\left\|X-Y_{n}^{N_{n}}\right\|_{2,-p, \mathbb{C}} \leq\left\|X-X_{n}\right\|_{2,-p, \mathbb{C}}+\left\|X_{n}-Y_{n}^{N_{n}}\right\|_{2,-p, \mathbb{C}}<\epsilon
$$

For $\mathbb{B}_{p}$-analyticity, we have the same result:

\section{COROLLARY 2.8}

If $\left\{X_{n}\right\}$ is a sequence of $\mathbb{B}_{p}$-analytic elements which converges to $X$ in $\left(L^{p}\right)$, then $X$ is $\mathbb{B}_{p}$-analytic.

\section{PROPOSITION 2.9}

If $X, Y$ are $\mathbb{B}$-analytic, then $X \diamond Y$ is $\mathbb{B}$-analytic.

\section{PROOF}

Let $\left\{X_{n}\right\}$ and $\left\{Y_{n}\right\}$ be two sequences of $\mathbb{B}$-analytic polynomials converging strongly in $\left(S_{\mathbb{C}}\right)^{-1}$ to $X$ and $Y$ respectively. That means, for $p, q>0$ :

$$
\left\|X_{n}-X\right\|_{2,-p, \mathbb{C}} \rightarrow 0, \quad n \rightarrow \infty
$$

and:

$$
\left\|Y_{n}-Y\right\|_{2,-q, \mathbb{C}} \rightarrow 0, \quad n \rightarrow \infty
$$

Define, for an $\alpha>\frac{1}{2}$ :

$$
r:=\alpha+\max (p, q)
$$


From the triangle inequality and Corollary 4.22 in [9], we have:

$$
\begin{aligned}
\| X & \diamond Y-X_{n} \diamond Y_{n}\left\|_{2,-r, \mathbb{C}} \leq\right\| X \diamond\left(Y-Y_{n}\right)\left\|_{2,-r, \mathbb{C}}+\right\| Y_{n} \diamond\left(X-X_{n}\right) \|_{2,-r, \mathbb{C}} \\
& \leq K_{1}\|X\|_{2,-p \mathbb{C}}\left\|Y-Y_{n}\right\|_{2,-q, \mathbb{C}}+K_{2}\left\|Y_{n}\right\|_{2,-q, \mathbb{C}}\left\|X-X_{n}\right\|_{2,-p, \mathbb{C}}
\end{aligned}
$$

We see that $X_{n} \diamond Y_{n}$ converges strongly to $X \diamond Y$, and since $X_{n} \diamond Y_{n}$ is a W-analytic polynomial, the proposition follows.

\section{THEOREM 2.10}

Let $p>1$ and $q>p$. Assume that $X \in\left(L^{q}\right)$ is $\mathbb{B}_{q}$-analytic and that $Y \in\left(L^{\frac{q p}{q-p}}\right)$ is $\mathbb{B} \frac{q p}{q-p}$-analytic. Then $X \cdot Y \in\left(L^{p}\right)$ is $\mathbb{B}_{p}$-analytic. Moreover,

$$
X \diamond Y=X \cdot Y
$$

\section{PROOF}

Let $X_{n}$ and $Y_{n}$ be the $\mathbb{B}$-analytic sequences converging to $X$ and $Y$ respectively. $X_{n} \cdot Y_{n}$ is of course again a $\mathbb{B}$-analytic polynomial. Observe that by the Cauchy-Schwartz inequality we have:

$$
\|f \cdot g\|_{p} \leq\|f\|_{q}\|g\|_{\frac{q p}{q-p}}
$$

Hence, by the triangle inequality it follows that:

$$
X_{n} \cdot Y_{n} \rightarrow X \cdot Y
$$

in $\left(L^{p}\right)$. Thus, $X \cdot Y$ is $\mathbb{B}_{p}$-analytic. Since $X_{n} \cdot Y_{n}$ is a $\mathbb{B}$-analytic polynomial, we have:

$$
X_{n} \cdot Y_{n}=X_{n} \diamond Y_{n}
$$

Since $X_{n}$ and $Y_{n}$ converge in $\left(L^{q}\right)$ and $\left(L^{\frac{q p}{q-p}}\right)$ respectively, we can show that:

$$
S\left(X_{n} \diamond Y_{n}\right)(\xi) \rightarrow S X(\xi) \cdot S Y(\xi)
$$

pointwise, and:

$$
\left|S\left(X_{n} \diamond Y_{n}\right)(\xi)\right| \leq K,
$$


uniformly in $n$. Here, $\xi$ is in a neighbourhood around zero in $S_{\mathbb{C}}(\mathbb{R})$. Hence, by Theorem 5 in [12], it follows that:

$$
X_{n} \diamond Y_{n} \rightarrow X \diamond Y
$$

weakly in $\left(S_{\mathbb{C}}\right)^{-1}$. By the corollary above, $X_{n} \cdot Y_{n} \rightarrow X \cdot Y$ strongly in $\left(S_{\mathbb{C}}\right)^{-1}$. Hence:

$$
X \diamond Y=\lim _{n} X_{n} \diamond Y_{n}=\lim _{n} X_{n} \cdot Y_{n}=X \cdot Y .
$$

where $\lim _{n}$ denotes the strong limit in $\left(S_{\mathbb{C}}\right)^{-1}$.

\section{Remark}

Note if $X$ is $\mathbb{B}_{q}$ analytic for some $q>1$ and $Y$ is $\mathbb{B}_{r}$-analytic for all $r<\infty$, then it is always possible to find $p>1$ s.t. the conditions in theorem 2.9 are satisfied.

We now go on to consider stochastic processes. Here we call a stochastic process $X_{t}$ $\mathbb{B}$-analytic if $X_{t}$ is $\mathbb{B}$-analytic for every fixed $t$ and similarly for $\mathbb{B}_{p}$-analyticity. We want to consider certain elementary observations on $\mathbb{B}$-analytic processes and start out with some observations.

\section{PROPOSITION 2.11}

Assume $X_{t}$ is an Itô integrable and that for each $t$ it can be approximated in $L^{2}$ by adapted $\mathbb{B}$-analytic polynomials. Then then Itô integral:

$$
\int_{0}^{T} X_{t} d \mathbb{B}_{t}
$$

is $\mathbb{B}_{2}$-analytic.

\section{PROOF}

By definition of the Itô integral:

$$
\int_{0}^{T} X_{t} d \mathbb{B}_{t}=\lim _{j} \sum_{j} X_{t_{j}} \cdot\left(\mathbb{B}_{t_{j+1}}-\mathbb{B}_{t_{j}}\right)
$$

where the limit is taken in $\left(L^{2}\right)$. By assumption, $X_{t_{j}}$ is $\mathbb{B}_{2}$-analytic. Since $X_{t}$ is adapted, we have:

$$
E\left[\left|X_{t_{j}}\right|^{2}\left|\mathbb{B}_{t_{j+1}}-\mathbb{B}_{t_{j}}\right|^{2}\right]=E\left[\left|X_{t_{j}}\right|^{2}\right] \cdot E\left[\left|\mathbb{B}_{t_{j+1}}-\mathbb{B}_{t_{j}}\right|^{2}\right] .
$$

Hence, we see that $X_{t_{j}}\left(\mathbb{B}_{t_{j+1}}-\mathbb{B}_{t_{j}}\right)$ is $\mathbb{B}_{2}$-analytic. The Itô integral is then the $\left(L^{2}\right)$-limit of $\mathbb{B}_{2}$-analytic elements, which imply the proposition. 


\section{PROPOSITION 2.12}

Let $\left\{x_{i}(t)\right\}_{i=1}^{\infty}$ be any sequence of $L^{2}(\mathbb{R})$-functions. Then all the integrals $\int x_{i}(t) d \mathbb{B}_{t}$, $i=1,2, \ldots$ are $\mathbb{B}_{q}$-analytic for any $q$ and any combination of Wick powers and Wick products of these random variables coincide with the corresponding expressions defined in terms of the ordinary product.

\section{PROOF}

It is an easy application of the Burkholder-Gundy inequalities to see that the integrals $\int x_{i}(t) d \mathbb{B}_{t}, i=1,2, \ldots$ are $\mathbb{B}_{q}$-analytic for any $q$. The second part follows from theorem 2.10 .

\section{PROPOSITION 2.13}

Let $X_{t}$ be a $\mathbb{B}$-analytic process where $\left\langle X_{t}, \phi\right\rangle$ is measurable on $[0, T]$ for all $\phi \in(S)^{1}$. Assume there exists a $p \geq 0$ such that:

$$
\int_{0}^{T}\left\|X_{t}\right\|_{2,-p, \mathbb{C}} d t<\infty
$$

Then the Bochner integral $\int_{0}^{T} X_{t} d t \in\left(S_{\mathbb{C}}\right)^{-1}$ is $\mathbb{B}$-analytic.

PROOF

By Pettis' Theorem (see e.g. [22]), the measurability of $\left\langle X_{t}, \phi\right\rangle$ implies strong measurability, i.e. the existence of a sequence $\left\{X_{t}^{n}\right\}_{n}$ in $\left(S_{\mathbb{C}}\right)^{-1}$ converging strongly to $X_{t}$. By inspection of the proof of Pettis' theorem in [22], this sequence can be chosen in the following manner:

$$
X_{s}^{n}=Y_{i}, \quad \text { when } s \in B_{i}^{n},
$$

where $\left\{B_{i}^{n}\right\}_{i=1}^{N_{n}}$ are disjoint measurable sets in $[0, T]$, and $Y_{i}=X_{s_{i}}$ for some $s_{i} \in[0, T]$. Hence, by assumption, $X_{t}^{n}$ is $\mathbb{B}$-analytic.

By the condition $\int_{0}^{T}\left\|X_{t}\right\|_{2,-p} d t<\infty$ Bochner integrability of $X_{t}$ follows. We have:

$$
\int_{0}^{T} X_{t} d t=\lim _{n \rightarrow \infty} \int_{0}^{T} X_{t}^{n} d t=\lim _{n \rightarrow \infty} \sum_{i=1}^{\infty} Y_{i} m\left(B_{i}^{n}\right) .
$$

The limit is strongly in $\left(S_{\mathbb{C}}\right)^{-1}$. Hence, the Bochner integral is the strong limit of $\mathbb{B}$-analytic elements, and the proposition follows. 
F.E.Benth et al

In the Kondratiev space we have a generalization of Itô/Skorohod integration. If $X_{t}$ is an Itô integrable process, then:

$$
\int_{0}^{T} X_{t} \diamond \mathbb{W}_{t} d t=\int_{0}^{T} X_{t} d \mathbb{B}_{t}
$$

We have the following result about $\mathbb{B}$-analyticity of this integral:

\section{PROPOSITION 2.14}

Let $X_{t}$ be a $\mathbb{B}$-analytic process such that:

i) $S X_{t}(\xi)$ is measurable for $\xi \in \mathcal{U}$

ii) $\left|S X_{t}(\xi)\right| \leq C(t)$, where $C(t) \in L^{1}([0, T], d t)$ for $\xi \in \mathcal{U}$

Then $X_{t} \diamond \mathbb{W}_{t}$ is Bochner integrable, and:

$$
\int_{0}^{T} X_{t} \diamond \mathbb{W}_{t} d t
$$

is $\mathbb{B}$-analytic.

PROOF

We have:

$$
\mathbb{W}_{t}=\lim _{h \rightarrow 0} \frac{1}{h}\left(\mathbb{B}_{t+h}-\mathbb{B}_{t}\right),
$$

where the limit is strong in $\left(S_{\mathbb{C}}\right)^{-1}$. Hence, $\mathbb{W}_{t}$ is a $\mathbb{B}$ - analytic process. This implies by proposition (above) that $X_{t} \diamond \mathbb{W}_{t}$ is $\mathbb{B}$-analytic. By Theorem 6 in [12], we have the Bochner integrability of $X_{t} \diamond \mathbb{W}_{t}$. Hence, the proposition follows.

\section{Applications to SDEs}

We now want to compare SDEs of the from:

$$
\begin{gathered}
d Z_{t}=\left(X_{t} \cdot Z_{t}+Y_{t}\right) d \mathbb{B}_{t}+\left(U_{t} \cdot Z_{t}+V_{t}\right) d t \\
d Z_{t}=\left(X_{t} \diamond Z_{t}+Y_{t}\right) d \mathbb{B}_{t}+\left(U_{t} \diamond Z_{t}+V_{t}\right) d t
\end{gathered}
$$

The equation (3.2) can be solved under very mild conditions on the coefficients. We will first consider the properties of this equation. If in addition, the coefficients are $\mathbb{B}$-analytic and sufficiently nice for (3.1) to make sense, we can expect the two solutions to coincide. 


\section{PROPOSITION 3.1}

Assume that for every $\xi \in S_{\mathbb{C}}(\mathbb{R})$, that the $S$-transforms $S\left(X_{t}\right)(\xi), S\left(Y_{t}\right)(\xi), S\left(U_{t}\right)(\xi)$ and $S\left(V_{t}\right)(\xi)$ are locally Lipschitz functions (as functions of $t$ ). If (3.2) has a $\left(S_{\mathbb{C}}\right)^{-1}$-valued solution defined for all $t \geq 0$, this solution is unique.

Remark: We call a function $f=f(z)$ locally Lipschitz if there for every $z_{0}$ exists a constant $C<\infty$ s.t. $\left|f(z)-f\left(z_{0}\right)\right| \leq C\left|z-z_{0}\right|$ for every $z$ in a neighbourhood of $z_{0}$.

\section{PROOF}

Apply the $S$-transform to both sides of (3.2) to see that that the $S$-transform of $Z_{t}$ is uniquely defined. Since any element in $\left(S_{\mathbb{C}}\right)^{-1}$ is uniquely defined in terms of its $S$ transform, the proposition follows.

\section{PROPOSITION 3.2}

Assume that:

i) $S\left(X_{t}\right)(\xi), S\left(Y_{t}\right)(\xi), S\left(U_{t}\right)(\xi)$ and $S\left(V_{t}\right)(\xi)$ are measurable for $\xi \in \mathcal{U}$

ii) $\left|S\left(X_{t}\right)(\xi)\right|,\left|S\left(Y_{t}\right)(\xi)\right|,\left|S\left(U_{t}\right)(\xi)\right|,\left|S\left(V_{t}\right)(\xi)\right| \leq C_{T}(t)$, where $e^{C_{T}(t)} \in L^{p}([0, T], d t)$ for every $\xi \in \mathcal{U}$, all $p>0$ and all $T<\infty$

iii) $Z_{0} \in\left(S_{\mathbb{C}}\right)^{-1}$ then (3.2) has a $\left(S_{\mathbb{C}}\right)^{-1}$-valued solution $Z_{t}$ given by the expression:

$$
\begin{aligned}
Z_{t} & =Z_{0} \diamond \operatorname{Exp}\left[\int_{0}^{t} X_{r} d \mathbb{B}_{r}+\int_{0}^{t} U_{r} d r\right] \\
& +\int_{0}^{t} \operatorname{Exp}\left[\int_{s}^{t} X_{r} d \mathbb{B}_{r}+\int_{s}^{t} U_{r} d r\right] \diamond Y_{s} d \mathbb{B}_{s} \\
& +\int_{0}^{t} \operatorname{Exp}\left[\int_{s}^{t} X_{r} d \mathbb{B}_{r}+\int_{s}^{t} U_{r} d r\right] \diamond V_{s} d s
\end{aligned}
$$

\section{PROOF}

The idea is to use the analogy with the differential equation $y^{\prime}=f(t) y+g(t)$. This equation has the solution $y=y_{0} e^{\int_{0}^{t} f(r) d r}+\int_{0}^{t} e^{\int_{s}^{t} f(r) d r} g(s) d s$. Formally we write that $f(t)=X_{t} \frac{d \mathbb{B}_{t}}{d t}+U_{t}$ and $g(t)=Y_{t} \frac{d \mathbb{B}_{t}}{d t}+V_{t}$. If we insert this in the solution formula and replace all the ordinary products with Wick products, we get (3.3). From the arguments of proposition 2.14, we see that all the necessary expressions are Bochner integrable and that (3.3) makes sense as an element of $\left(S_{\mathbb{C}}\right)^{-1}$. If we insert this expression in (3.2) all the operations in (3.2) are well defined. Hence the ordinary chain rule applies and $Z_{t}$ is a solution. 
As we remarked earlier the interesting question is to compare the equation (3.1) and (3.2). To make sense out of (3.1) we must put quite strong growth conditions on the coefficients. We have to work with elements in $\left(L^{p}\right)$. The idea is then to apply theorem 2.10 to see that the Wick products coincide with the ordinary product.

\section{THEOREM 3.3}

Assume that:

i) $X_{t}=x(t)$ where $x(t) \in L^{2}(\mathbb{R})$

ii) $U_{t}=\int_{0}^{t} u_{1}(s) d \mathbb{B}_{s}+u_{2}(t)$ where $u_{1}(t), u_{2}(t) \in L^{2}(\mathbb{R})$

iii) $Y_{t}, V_{t}$ are adapted and $\mathbb{B}_{q}$-analytic for some $q>2$ and satisfies the conditions in 3.2.

iv) $Z_{0}$ is $\mathbb{B}_{r}$-analytic for some $r>1$ (If $Z_{0}$ is non-constant, the meaning of (3.1) is interpreted in the Hitsuda-Skorohod sense, se [8]).

Then the solutions of (3.1) and (3.2) coincide and are both given by the expression:

$$
Z_{t}=Z_{0} \cdot e^{\int_{0}^{t} X_{r} d \mathbb{B}_{r}+\int_{0}^{t} U_{r} d r}+\int_{0}^{t} e^{\int_{s}^{t} X_{r} d \mathbb{B}_{r}+\int_{s}^{t} U_{r} d r} \cdot Y_{s} d \mathbb{B}_{s}+\int_{0}^{t} e^{\int_{s}^{t} X_{r} d \mathbb{B}_{r}+\int_{s}^{t} U_{r} d r} \cdot V_{s} d s
$$

Before we turn to the proof of this theorem, we need to prove two technical lemmas.

\section{LEMMA 3.4}

If $e^{|X|} \in\left(L^{p}\right)$ for all $p$ and $X$ is $\mathbb{B}_{p}$-analytic for all $p$, then $e^{X}$ is also $\mathbb{B}_{p}$-analytic for all $p$.

\section{PROOF}

Put $f_{N}=e^{|X|}-\sum_{n=0}^{N} \frac{1}{n !}|X|^{n}$. By the monotone convergence theorem $f_{N} \rightarrow 0$ in every $\left(L^{p}\right)$. Observe that:

$$
\mathrm{E}\left[\left|e^{X}-\sum_{n=0}^{N} \frac{1}{n !} X^{n}\right|^{p}\right]=\mathrm{E}\left[\left|\sum_{n=N+1}^{\infty} \frac{1}{n !} X^{n}\right|^{p}\right] \leq \mathrm{E}\left[\left.\left.\left|\sum_{n=N+1}^{\infty} \frac{1}{n !}\right| X\right|^{n}\right|^{p}\right]=\mathrm{E}\left[\left|f_{N}\right|^{p}\right] \rightarrow 0
$$

Hence $e^{X}$ can be approximated as well as we please by $\sum_{n=0}^{N} \frac{1}{n !} X^{n}$ in any $\left(L^{p}\right)$. Since $X$ is $\mathbb{B}_{q}$-analytic for every $q$, clearly each $\sum_{n=0}^{N} \frac{1}{n !} X^{n}$ is $\mathbb{B}_{p}$-analytic and this proves the lemma. 


\section{LEMMA 3.5}

$e^{\int_{0}^{t} X_{r} d \mathbb{B}_{r}}$ and $e^{\int_{0}^{t} U_{r} d r}$ are adapted and $\mathbb{B}_{p}$-analytic for every $p$.

\section{PROOF}

The adaptedness is trivial. Fix $t$ and put $X=\int_{0}^{t} x(s) d \mathbb{B}_{r}$. By proposition $2.12 X$ is $\mathbb{B}_{q^{-}}$ analytic for every $q$. Choose any $p$. We must prove that $e^{|X|} \in\left(L^{p}\right)$. For simplicity we will replace $x$ and $\mathbb{B}$ by the corresponding real expressions in the rest of the argument. Since $X$ is gaussian, we then have:

$$
\mathrm{E}\left[|X|^{2 n}\right]=\frac{(2 n) ! \mathrm{E}\left[|X|^{2}\right]}{2^{n} n !}=\frac{(2 n) ! \int_{0}^{t} x(s)^{2} d s}{2^{n} n !}
$$

From this we get using Stirlings formula:

$$
\begin{aligned}
\mathrm{E}\left[e^{p|X|}\right] & =\sum_{n=0}^{\infty} \frac{p^{n}}{n !} \mathrm{E}\left[|X|^{n}\right] \leq \sum_{n=0}^{\infty} \frac{p^{n}}{n !}\left(\mathrm{E}\left[|X|^{2 n}\right]\right)^{\frac{1}{2}} \\
& =\sum_{n=0}^{\infty} \frac{p^{n}}{n !}\left(\frac{(2 n) ! \int_{0}^{t} x(s)^{2} d s}{2^{n} n !}\right)^{\frac{1}{2}}<\infty
\end{aligned}
$$

Hence $X$ satisfies the hypothesis of the previous lemma and the conclusion follows. The proof for the expression $e^{\int_{0}^{t} U_{r} d r}$ is similar.

We now turn to the proof of theorem 3.4.

PROOF

We first want to prove that the expression in (3.3) equals the expression in (3.4). Since $\int_{0}^{t} X_{r} d \mathbb{B}_{r}$ and $\int_{0}^{t} U_{r} d r$ are $\mathbb{B}_{q}$-analytic for every $q$, we have:

$$
\operatorname{Exp}\left[\int_{s}^{t} X_{r} d \mathbb{B}_{r}+\int_{s}^{t} U_{r} d r\right]=e^{\int_{s}^{t} X_{r} d \mathbb{B}_{r}+\int_{s}^{t} U_{r} d r}
$$

It then follows by lemma 3.6, theorem 2.10 and proposition 2.11 that:

$$
\int_{0}^{t} \operatorname{Exp}\left[\int_{s}^{t} X_{r} d \mathbb{B}_{r}+\int_{s}^{t} U_{r} d r\right] \diamond Y_{s} d s=\int_{0}^{t} e^{\int_{s}^{t} X_{r} d \mathbb{B}_{r}+\int_{s}^{t} U_{r} d r} \cdot Y_{s} d s
$$

The same arguments works to prove that all the terms in (3.3) and (3.4) are equal. If we insert the expression (3.4) on the right side of (3.1), we may replace all the the ordinary products by Wick products. Hence the right side of (3.1) equals $d Z_{t}$ and this completes the proof of theorem 3.4. 
F.E.Benth et al

LEMMA 3.6

Let $f$ be analytic in a neighbourhood of $y_{0}$. Then the differential equation:

$$
y^{\prime}=f(y) \quad y\left(z_{0}\right)=y_{0}
$$

has a unique solution $y=y(z)$ analytic in a neighbourhood of $z_{0}$.

\section{PROOF}

Local uniqueness follows from the Lipschitz continuity of $f$ at $y_{0}$. If $f\left(y_{0}\right)=0$, then $y(z)=y_{0}$ is the solution. If on the other hand $f\left(y_{0}\right) \neq 0$, then $f(z) \neq 0$ in a neighbourhood of $y_{0}$ and the function $\frac{1}{f(z)}$ is analytic on this neighbourhood. Hence there exists a neighbourhood of $y_{0}$ and an analytic function $g(z)$ on this neighbourhood s.t. $g^{\prime}(z)=\frac{1}{f(z)}$. Since $g^{\prime}(z) \neq 0$ it follows from the inverse function theorem, see [16] theorem 1.3.7, that $g$ has an inverse function $h$ which is analytic in some neighbourhood of $g\left(y_{0}\right)$. Put $y(z)=h\left(z-z_{0}+g\left(y_{0}\right)\right)$. Then $y\left(z_{0}\right)=y_{0}$ and:

$$
\begin{aligned}
y^{\prime} & =h^{\prime}\left(z-z_{0}+g\left(y_{0}\right)\right)=h^{\prime}\left(g\left(h\left(z-z_{0}+g\left(y_{0}\right)\right)\right)\right. \\
& =\frac{1}{g^{\prime}\left(h\left(z-z_{0}+g\left(y_{0}\right)\right)\right.}=f\left(h\left(z-z_{0}+g\left(y_{0}\right)\right)=f(y)\right.
\end{aligned}
$$

Hence $y=y(z)$ is analytic in a neighbourhood of $z_{0}$ and is a solution of (3.10).

\section{THEOREM 3.7}

Let $f$ be analytic in a neighbourhood $D$ and let $z_{0} \in D$. Then:

$$
d Z_{t}=f^{\diamond}\left(Z_{t}\right) d \mathbb{B}_{t} \quad Z_{0}=z_{0}
$$

has a unique $\left(S_{\mathbb{C}}\right)^{-1}$-valued solution $Z_{t}$ defined for all $t \geq 0$. Moreover there exists a stopping time $\tau>0$ s.t. $Z_{t \wedge \tau}$ is a local solution to:

$$
d Z_{t}=f\left(Z_{t}\right) d \mathbb{B}_{t} \quad Z_{0}=z_{0}
$$

\section{PROOF}

Uniqueness. Choose and fix $\xi \in S_{\mathbb{C}}(\mathbb{R})$. Then apply the $S$-transform to both sides of (3.12) to see that $y=S\left(Z_{t}\right)(\xi)$ is a solution to the ODE:

$$
y^{\prime}=f(y) \bar{\xi}(t) \quad y(0)=z_{0}
$$


Since (3.14) has a unique solution $y$ and the $S$-transform uniquely characterizes every element in $\left(S_{\mathbb{C}}\right)^{-1}$, the solution $Z_{t}$ of (3.12) is unique.

Existence. First use the previous lemma to find an analytic function $y(z)=\sum_{k=0}^{\infty} a_{k} z^{k}$ s.t. $y$ is a solution to the problem:

$$
y^{\prime}=f(y) \quad y(0)=z_{0}
$$

Since $y$ is analytic in a neighbourhood of the origin, there exists two positive constants $M<\infty, r<\infty$ s.t. $\left|a_{k}\right| \leq M r^{k}$. The expression:

$$
Z_{t}=\sum_{k=0}^{\infty} a_{k} \mathbb{B}_{t}^{\diamond k}=\sum_{k=0}^{\infty} a_{k} \mathbb{B}_{t}^{k}
$$

then makes sense as an element of $\left(S_{\mathbb{C}}\right)^{-1}$. The ordinary chain rule applies, and hence $Z_{t}$ is a solution of (3.12). Now let $\tau_{d}$ be the first exit time of $\mathbb{B}_{t}$ from a small neighbourhood of the origin. If we put $Y_{t}=Z_{t \wedge \tau_{d}}=\sum_{k=0}^{\infty} a_{k} \mathbb{B}_{t \wedge \tau_{d}}^{k}$, it follows from the complex Ito formula that the ordinary chain rule applies. $Y_{t}$ is then a local solution to (3.13) in the sense that it solves the problem $Z_{t \wedge \tau_{d}}=z_{0}+\int_{0}^{t \wedge \tau_{d}} f\left(Z_{s}\right) d \mathbb{B}_{s}$.

\section{ACKNOWLEDGEMENT}

This work is supported by VISTA, a research cooperation between The Norwegian Academy of Science and Letters and Den Norske Stats Oljeselskap (Statoil).

The first author also wishes to express his gratitude to Norges Forskningsråd for the support by NAVF grant 100549/410.

\section{REFERENCES}

1. S.Albeverio, Y.L.Daletsky, Y.G.Kondratiev, and L.Streit (1994). Non-Gaussian Infinite Dimensional Analysis. Preprint nr. 217, University of Bochum, Germany.

2. H.Arai (1986). On the algebra of bounded holomorphic martingales. Proc.Amer. Math.Soc. 97, 616-620.

3. F.E.Benth (1993). Integrals in the Hida distribution space $(S)^{*}$. T. Lindstrøm, B.Øksendal and A.S.Ustunel (editors): Stochastic Analysis and Related Topics, Gordon \& Breach, 89-99.

4. M.Fukushima and M.Okada (1984). On conformal martingale diffusions and pluripolar sets. J.Func.Anal. 55, 377-388.

5. R.K.Getoor and M.J.Sharpe (1972). Conformal martingales. Invent.Math. 16, 271-308.

6. H.Gjessing, H.Holden, T.Lindstrøm, B.Øksendal, J.Ubøe and T.-S.Zhang (1993). Wick multiplication, H.Niemi et al. (editors): Frontiers in Pure and Applied Probability, TVP Publishers, Moskow, 29-67.

7. T.Hida (1980), Brownian Motion, Springer. 
8. T.Hida, H.-H.Kuo, J.Potthoff and L.Streit (1993). White Noise: An infinite dimensional calculus, Mathematics and its applications 253, Kluwer.

9. H.Holden, T.Lindstrøm, B.Øksendal, J.Ubøe and T.-S.Zhang (1993). Stochastic boundary value problems. A white noise functional approach", Probability Theory and Related Fields 95, 391-419.

10. H.Holden, T.Lindstrøm, B.Øksendal, J.Ubøe and T.-S.Zhang (1995). Stochastic partial differential equations - White Noise Functional Methods, Models and Applications, book manuscript.

11. H-H.Kuo (1992). Lectures on white noise analysis, Soochow J.Math. 18, no.3, 229-300.

12. Y.G.Kondratiev, P.Leukert and L.Streit (1994). Wick Calculus in Gaussian analysis. Preprint nr. 637/6/94, University of Bielefeld, Germany.

13. T. Lindstrøm, B. Øksendal and J. Ubøe (1992). Wick multiplication and Ito-Skorohod stochastic differential equations. In S. Albeverio et al (editors): Ideas and Methods in Mathematical Analysis, Stochastics, and Applications. Cambridge Univ. Press, 183206.

14. B.Øksendal (1992). Stochastic Differential Equations, 3 edn., Springer Verlag.

15. J.Potthoff and L.Streit (1991). A characterization of Hida distributions, J.Func. Anal. $101,212-229$.

16. W.Rudin (1980). Function Theory in the Unit Ball of $\mathbb{C}^{n}$, Springer.

17. L.Schwartz (1980). Semi-martingales sur des variétés, et martingales conformes sur des variétés analytiques complexes. Lecture notes in Mathematics No.780, Springer Verlag.

18. J.Ubøe (1987). Conformal martingales and analytic functions. Math.Scand. 60, 292309.

19. J.Ubøe (1992). Riemann integration on complex brownian paths. Stoch.Anal.Appl. 10(3), 351-361.

20. J.Ubøe (1994). Complex valued, multiparameter stochastic integrals. To appear in J.Theor.Prob..

21. M.Yor (1977). Étude de certains processus (stochastiquement) différentiables ou holomorphes. Ann.Inst.Henri Poincare XIII No.1, 1-25.

22. K.Yosida (1978). Functional Analysis, Springer.

23. T.-S.Zhang (1992). Characterizations of white noise test functions and Hida distributions, Stochastics 41, 71-87. 